\title{
The Antarctic Planet Interferometer
}

\author{
Mark R. Swain \\ Jet Propulsion Laboratory, Pasadena, CA
}

\begin{abstract}
.
The Antarctic Planet Interferometer (API) is a concept for an infrared interferometer located at the best accessible site on Earth. Infrared interferometry is strongly effected by both the strength and vertical distribution of thermal and water vapor turbulence. The combination of low temperature, low wind speed, low elevation turbulence, and low precipitable water vapor make the Concordia base at Antarctic Dome $\mathrm{C}$ the best accessible site on Earth for infrared interferometry. The improvements in interferometer sensitivity with respect to other terrestrial sites are dramatic; an interferometer with two meter class telescopes could make unique infrared measurements of extra solar planets that might otherwise only be possible with a space-based interferometer.
\end{abstract}

\section{Unique Science from Earth; A Stepping Stone to Space}

A number of spatial interferometry techniques are being developed for measurements of extra-solar planets including high-precision $V^{2}$, differential-phase, nulling, and astrometry. The unique properties of the Antarctic atmosphere at Dome C likely improve all of these techniques, compared to other terrestrial sites. Estimates for the performance gains for infrared interferometry at Dome C (Swain et al. 2003a) and at the South Pole (Lloyd et al. 2002, 2003) imply order of magnitude or better improvements are possible. These estimates are based on extensive site testing data including measurements of the turbulence profile (Marks et al. 1996, 1999; Marks 2002; Travouillon et al. 2003).

In addition to unique measurements of extra-solar planets, such as characterization of Jovian-class extra-solar planets in the habitable zone, an infrared Antarctic interferometer would be capable of other ambitious science programs (Swain et al. 2003a) such as:

- Distance scale - measurement of binary star orbits in LMC.

- Mass transfer in compact binary systems.

- YSO and proto-planetary disk formation.

- Active galactic nuclei.

- Accretion disk structure and evolution.

Recent observations with the Keck interferometer (Swain et al. 2003b) have marginally resolved what is likely infrared emission from the accretion disk in NGC 4151. Modest improvements in sensitivity and angular resolution, easily 
achievable with API, would allow measurements of the structure and evolution of the accretion disk in similar objects.

There are additional advantages of an Antarctic plateau location. The long night results in good instrument thermal stability. Most of the sources observed would be circumpolar, allowing extremely long, continuous observations not possible from other locations. These sources would also be excellent targets for interferometric synthesis imaging as they permit full rotation of the interferometer sampling function.

Further, because of similar science goals to projects such as DARWIN and TPF and a space-like environment (due to the extreme temperatures and remote location), the API, located at the Concordia base, could serve as a natural technology development test bed for these missions. When operating at 200 $\mu \mathrm{m}$, API would also be an excellent prototype for the proposed SPECS mission (Leisawitz et al. 2000). In addition to producing unique and compelling science, an Antarctic infrared interferometer would serve as a precursor for future spacebased interferometers.

\section{Acknowledgments}

This work was performed at the Jet Propulsion Laboratory, California Institute of Technology, under a contract with the National Aeronautics and Space Administration.

\section{References}

Leisawitz, D. T., et al. 2000, Proc. SPIE, 4013, 36

Lloyd, J. P., Lane, B. F., Swain, M. R., Storey, J. W. V., Travouillon, T., Traub, W. A., \& Walker, C. K. 2003, in Techniques and Instrumentation for Detection of Exoplanets, proc. SPIE 5170, 193

Lloyd, J. P., Oppenheimer, B. R., \& Graham, J. R. 2002, Publications of the Astronomical Society of Australia, 19, 318

Marks, R. D. 2002, A\&A, 385, 328

Marks, R. D., Vernin, J., Azouit, M., Briggs, J. W., Burton, M. G., Ashley, M. C. B., \& Manigault, J. F. 1996, A\&AS, 118, 385

Marks, R. D., Vernin, J., Azouit, M., Manigault, J. F., \& Clevelin, C. 1999, A\&AS, 134,161

Swain, M. R., Coude du Foresto, V., Fossat, E., Vakili, F. 2003a, Mem. S.A. It.,73, 23.

Swain, M. R., et al. 2003b, ApJ, 596, 163

Travouillon, T., Ashley, M. C. B., Burton, M. G., Storey, J. W. V., \& Loewenstein, R. F. 2003, A\&A, 400, 1163 